\title{
Optimal micro channel recuperators for small-sized gas turbines
}

\author{
Martine Baelmans; Frederik Rogiers; Joris Van Eyndhoven \& Willem Voets \\ Katholieke Universiteit Leuven, Dept. Mechanical Engineering, Celestijnenlaan 300A, B-3001 \\ Leuven-Heverlee, Belgium
}

\begin{abstract}
Thermodynamic efficiencies based on the first and second law of thermodynamics are assessed for their use in the design of recuperators in small-sized gas turbines. It is shown that the first and second law cycle efficiency as well as the Witte-Shamsundar efficiency [1] for heat exchangers show a similar dependency on the heat exchanger pressure drops. This results in design relations for hydraulic diameter ratios and their related number of channels ratio. Further, it is shown that the WitteShamsundar efficiency focusing on heat exchanger inlet temperatures only can be used as a good approximation for the overall optimal recuperator design when also pressure drops over the recuperator are accounted for. However the approximation deteriorates with higher heat exchanger mass flow density and lower compressor ratio as well as with decreasing turbine inlet temperature ratio. Deviations from the full cycle efficiency considerations are assessed. It is concluded that the use of the WitteShamsundar efficiency for optimization of recuperators in small-sized applications like the $1.5 \mathrm{~kW}$ PowerMems gas turbine needs careful assessment.
\end{abstract}

\section{INTRODUCTION}

The current trend in miniaturizing energy conversion systems such as gas turbines and fuel cells together with the promising potential of micro process technologies has led to an increased interest in miniature heat exchangers [2-4]. As such, driven by its effective use in standard gas turbines heat recuperation is explored to enhance the overall cycle efficiency of small sized gas turbines. In this case, this improvement is even more crucial to compensate for the already low cycle efficiency due to significant lower achievable compressor pressure ratios and turbine inlet temperatures. It is clear that high heat exchanger effectiveness together with low pressure drops is favorable to achieve maximal cycle efficiency. However, effectiveness and pressure drops are inherently coupled via the recuperator configuration. Further, the effect of these parameters increases with decreasing recuperator channel dimensions. In addition, due to the smaller compressor pressure ratios and reduced turbine inlet temperatures in small sizes gas turbines, pressure drops in the recuperator can significantly reduce the cycle efficiency. Finding a compromise between these conflicting requirements within a certain volume is the main challenge in recuperator design.

Micro gas turbines in the range of 50-200 kW have found acceptance in large quantities in distributed power generation and become increasingly important. For this type of small gas turbines, heat recuperation is mandatory in order to achieve thermal efficiencies in the order of $30 \%$. Several micro recuperators in this range of power output are already developed [5,6]. Hereby focus is especially put on technological requirements and low cost fabrication technology rather than on optimization for achieving maximal cycle efficiency [7]. Also at smaller scales, for micro gas turbines in the power range smaller than $3 \mathrm{~kW}$, several micro recuperators are described in literature [8-10]. Thus far, almost no attention is paid to the generalization of these concepts in specific design rules. Stevens et al. [11] mentioned first general design requirements for recuperators in micro gas turbines and concluded that pressure drops are preferably located at the cold side of the recuperator. Moreover Stevens and Baelmans [12] deduced relations in design parameters for micro channel heat exchangers in gas turbine cycles.

In this paper the use of cost functions for the optimization of recuperators in micro gas turbines is assessed. Hereby, it is investigated whether the WitteShamsundar efficiency computed based on heat exchanger inlet parameters only can serve as a cost function for this optimization problem. In a first section the use of different cost functions is discussed and their influencing parameters are introduced. Next, the theoretical background for optimal micro channel heat exchanger design is presented, based on design parameter reduction as described in [12]. In a third section the optimal heat exchanger designs using the different cost functions are discussed and the deviation of the Witte-Shamsundar in comparison to full optimization is assessed with respect to mass flow density at one hand and non-dimensional cycle parameters on the other. The application case is a $1.5 \mathrm{~kW}$ micro gas turbine as developed in the PowerMEMS project $[3,4]$. 


\section{Optimal micro channel recuperators for small sized gas turbines, M. Baelmans et al.}

\section{COST FUNCTIONS FOR CYCLE OPTIMIZATION}

\subsection{The Thermodynamic Brayton Cycle}

In order to achieve an optimized recuperator design for small-sized gas turbines, it is evident that pressure drops have to be incorporated whenever the cycle efficiency is studied. This leads for a Brayton cycle with heat recuperation to the thermodynamic representation as shown in figure 1.

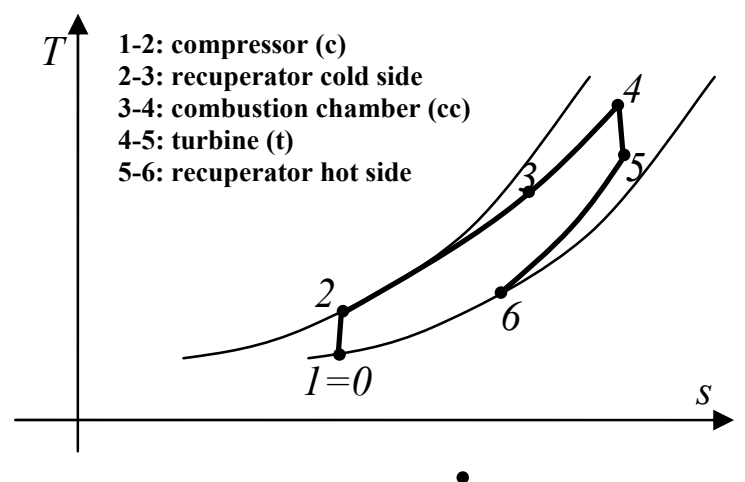

Figure 1. Thermodynamic states for a Brayton cycle with heat recuperation

The different states are defined by the associated thermodynamic parameters: pressure ratios $\pi=p_{\text {out }} / p_{\text {in }}$ over the individual components, compressor and turbine efficiencies (resp. $\eta_{c}$ and $\eta_{t}$ ), turbine inlet temperature $T_{4}$, recuperator effectiveness $\varepsilon$ and atmospheric pressure and temperature, resp. $p_{0}$ and $T_{0}$.

The temperatures of the different states can be easily computed from these parameters:

$$
\begin{aligned}
& \frac{T_{2}}{T_{0}}=\alpha_{c}=1-\frac{1-\pi_{c}^{\frac{\kappa-1}{\kappa}}}{\eta_{c}} \\
& \frac{T_{3}}{T_{0}}=\alpha_{c}+\varepsilon\left(\tau \alpha_{t}-\alpha_{c}\right) \\
& \frac{T_{4}}{T_{0}}=\tau \\
& \frac{T_{5}}{T_{0}}=\tau \alpha_{t}=\tau\left(1-\eta_{t}\left(1-\pi_{t}^{\frac{\kappa-1}{\kappa}}\right)\right) \\
& \frac{T_{6}}{T_{0}}=\tau \alpha_{t}(1-\varepsilon)+\varepsilon \alpha_{c}
\end{aligned}
$$

with $\pi_{c}$ a fixed compressor ratio and the turbine pressure ratio $\pi_{t}$ affected by all pressure drops in the cycle:

$$
\pi_{t}=\left(\pi_{c} \pi_{c c} \pi_{o} \pi_{\Delta p_{c}} \pi_{\Delta p_{h}}\right)^{-1} .
$$

In the right hand side of Equation (2.2) respectively the pressure ratios over compressor, combustion chamber, outlet, and cold and hot side of the recuperator are used. It should be noted that as such the influence of recuperator pressure drops to Expression (2.1) is incorporated in the variable $\alpha_{t}$ via $\pi_{t}$ only, whereas the heat exchanger effectiveness $\varepsilon$ is explicitly retained in expressions (2.1).

The term $\pi_{t}^{\frac{\kappa-1}{\kappa}}$ can be further elaborated with respect to the pressure drops in the recuperator at hot and cold side (resp. $\Delta p_{h}$ and $\Delta p_{c}$ ). Indeed, for the non-dimensional pressure drop at the cold side $\Delta p_{c}^{*} \equiv \frac{\Delta p_{c}}{p_{0} \pi_{c}}<<1$ and for the outlet pressure drop $\Delta p_{o}<<p_{0}$, which is most often the case for optimal recuperator design,

$$
\begin{aligned}
\pi_{\Delta p_{c}} \pi_{\Delta p_{h}} & =\left(1-\frac{\Delta p_{c}}{\pi_{c} p_{0}}\right)\left(1+\frac{\Delta p_{h}}{p_{0}+\Delta p_{o}}\right)^{-1} \\
& \approx 1-\frac{\Delta p_{c}+\pi_{c} \Delta p_{h}}{p_{0} \pi_{c}}=1-\pi_{\Delta p}^{*}
\end{aligned}
$$

with $\pi_{\Delta p}^{*} \equiv \frac{\Delta p_{c}+\pi_{c} \Delta p_{h}}{p_{0} \pi_{c}}$ the non-dimensional total pressure drop.

Based on Eq (2.3), $\pi_{t}{ }^{\frac{\kappa-1}{\kappa}}$ can be rewritten as:

$$
\pi_{t}^{\frac{\kappa-1}{\kappa}}=\frac{1+\frac{\kappa-1}{\kappa} \frac{\Delta p_{c}+\pi_{c} \Delta p_{h}}{p_{0} \pi_{c}}}{\left(\pi_{c} \pi_{c c} \pi_{o}\right)^{\frac{\kappa-1}{\kappa}}}
$$

Thus, the effect of the recuperator pressure drops is retrieved in the expressions for the temperatures by $\alpha_{t}$ as a function of the non-dimensional total pressure drop only.

\subsection{Cost Functions for Optimization}

The most straightforward candidate for cycle optimization is the cycle efficiency based on the first law of thermodynamics. Neglecting temperature dependence of $c_{p}$ and keeping the mass flow through the components constant, this leads to:

$$
\eta_{I}=\frac{T_{4}-T_{5}-T_{2}+T_{0}}{T_{4}-T_{3}}=\frac{1+\tau-\alpha_{c}-\tau \alpha_{t}}{\tau-(1-\varepsilon) \alpha_{c}-\varepsilon \tau \alpha_{t}} .
$$

Equally well the cost function can be based on the cycle efficiency based on the second law of thermodynamics. To this end, the system is considered from the point of view that heat per unit mass flow $q$ is added to the cycle at a boundary temperature $T_{b}$ and taking account for the exergy leaving the system at the outlet:

$$
\eta_{I I}=1-\frac{-q \frac{T_{0}}{T_{b}}+c_{p}\left(T_{6}-T_{0}\right)}{q \frac{T_{b}-T_{0}}{T_{b}}} .
$$




\section{Optimal micro channel recuperators for small sized gas turbines, M. Baelmans et al.}

Maximizing $\eta_{I I}$ results in minimizing the second term of the RHS in (2.6). Moreover for optimization the exact value for $T_{b}$ can be arbitrarily chosen depending on the systems boundary. Thus, for optimization this value can be virtually set to $\infty$. As such, the cost function to be maximized becomes exactly equal to the first law cycle efficiency as $\eta_{I}=\frac{l_{\text {net }}}{q}$ and $\eta_{I I}=\frac{l_{\text {net }}}{q\left(\frac{T_{b}-T_{0}}{T_{b}}\right)}$, with $l_{\text {net }}$ the net power delivered by the cycle. Thus, both cost functions exhibit a combined influence from cycle parameters $\tau, \alpha_{c}$ and $\eta_{t}\left(1-\left(\pi_{c} \pi_{c c} \pi_{o}\right)^{\frac{\kappa-1}{\kappa}}\right)$ at one hand and on the recuperator parameters $\varepsilon$ and $\pi_{\Delta p}^{*}$ on the other. The question now rises whether the Witte-Shamsundar efficiency can equally well be used to design an optimal small sized recuperator. The latter efficiency is based on the inlet temperatures of the recuperator and is defined in its simplest form as [1]:

$\eta_{W S}=1-\frac{\dot{I}_{H E X}}{\dot{Q}_{H E X}} \approx 1-\frac{T_{0} \ln \left(\frac{T_{3}}{T_{2}} \frac{T_{6}}{T_{5}}\right)}{T_{3}-T_{2}}$

Or taking account for pressure drops in the recuperator:

$\eta_{W S}^{*}=1-\frac{\dot{I}_{H E X}}{\dot{Q}_{H E X}}=1-\frac{T_{0}\left[\ln \left(\frac{T_{3}}{T_{2}} \frac{T_{6}}{T_{5}}\right)-\frac{\kappa-1}{\kappa} \ln \left(\pi_{\Delta p_{c}} \pi_{\Delta p_{h}}\right)\right]}{T_{3}-T_{2}}$

It should be noted that both expression (2.8) and (2.9) can become negative. This is possible for cryogenic operational conditions, where it might lead to inconvenient results [13]. However, within the application of Brayton cycles the cold inlet is close to environmental temperature leading to positive values for this efficiency. Further, though most often the second term in the numerator of (2.9) is neglected [1,13,14]; it should be retained in this case as pressure drop is a crucial parameter for the optimization of recuperators for small sized gas turbines. This second term can again be rewritten as a function of $\pi_{\Delta p}^{*}$.

Using equations (2.1), $\eta_{W S}$ and $\eta_{W S}^{*}$ can be reformulated with respect to the same cycle and heat exchanger parameters as for the previous cost functions. However, when used as such, the use of the Witte-Shamsundar efficiency would not give any advantage over the evaluation of the cost function based on the cycle efficiency. Indeed, in this case the recuperator inlet temperatures needed are still depending on cycle parameters including the pressure drops over the recuperator. Therefore, a more useful cost function would be to use $\eta_{W S, 0}^{*}$, similarly defined as $\eta_{W S}^{*}$ but with recuperator inlet temperatures defined based on zero pressure drops over the recuperator.

\section{HEAT EXCHANGER MODELING AND OPTIMIZATION PROCEDURE}

In the previous section the cost functions for evaluating the gas turbine recuperator were discussed. In order to further proceed with the analysis, information on the heat exchanger model is essential. Therefore in this section a model for micro channel heat exchangers is presented. This model can also be used for plate heat exchangers. Further, this model is combined with the cycle cost functions described in the previous section. Thus optimization relations can be deduced.

\subsection{Heat Exchanger Model}

The counter-flow heat exchanger under investigation consists of alternating layers in which micro channels for hot and cold fluid flows are produced and is depicted in Figure 2. For the present derivations the channels are assumed to be square and no wall thickness between the channels is taken into account. The heat exchanger has a total cross-sectional rectangular shape with area $A$. This leads to the following constraints:

$n_{c} D_{h, c}=n_{h} D_{h, h}$ and $n_{c} D_{h, c}^{2}+n_{h} D_{h, h}^{2}=A$

With $n_{i}$ the number of channels, $D_{h, i}$ the hydraulic diameter and $i=c$ or $i=h$ for cold and hot channels respectively.

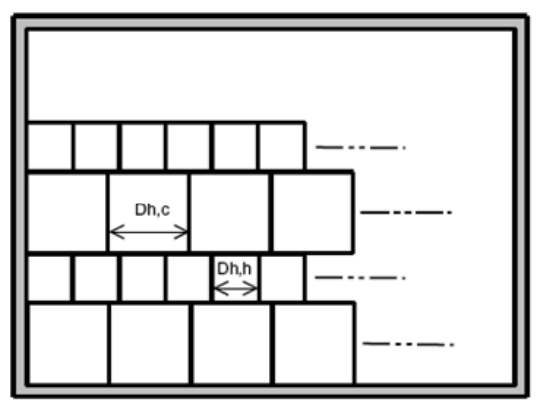

Figure 2. Heat exchanger configuration [12]

The pressure drop $\Delta p$ over each side of the heat exchanger equals:

$$
\Delta p=\frac{m^{\prime \prime} f \operatorname{Re} \mu L}{2 n \rho D_{h}^{4}}
$$

with $m^{\prime \prime}=\frac{\dot{m}}{A}$ the mass flux density, $f$ the Darcy friction factor, $R e$ the Reynolds number based on $D_{h}$ and $f R e=56.92, \mu$ the fluid viscosity, $L$ the channel length and $\rho$ the fluid density. Further $\varepsilon$ is determined by:

$$
\varepsilon=\frac{N T U}{1+N T U}
$$




\section{Optimal micro channel recuperators for small sized gas turbines, M. Baelmans et al.}

with

$$
N T U=\left(R_{t o t} \dot{m} c_{p}\right)^{-1}=\left(\frac{\dot{m} c_{p}}{4 L N u k}\left(\frac{1}{n_{c}}+\frac{1}{n_{h}}\right)\right)^{-1}
$$

Starting from Equations (3.1) and (3.2) explicit expressions for $n$ can be obtained. Substituting these expressions in Equations (3.4) and (3.3) leads to [12]:

$$
\begin{aligned}
& \varepsilon=\left[1+\frac{m^{\prime \prime} f \operatorname{RePr}}{8 N u}\left(\frac{1}{\rho_{c} \Delta p_{c}}\left(1+\left(\frac{\rho_{c} \Delta p_{c}}{\rho_{h} \Delta p_{h}}\right)^{\frac{1}{3}}\right)^{2}\right.\right. \\
& \left.\left.+\frac{1}{\rho_{h} \Delta p_{h}}\left(1+\left(\frac{\rho_{h} \Delta p_{h}}{\rho_{c} \Delta p_{c}}\right)^{\frac{1}{3}}\right)^{2}\right)\right]^{-1}
\end{aligned}
$$

\subsection{Optimal Heat Exchanger Design}

As shown in [12], the cycle efficiency $\eta_{I}$ is governed by the hot and cold channel pressure drops and the cycle parameters: it shows a linear dependence on both variables as depicted in Figure 3 (in this figure isoefficiency lines are plotted with dashed lines). This feature is due to the fact that the pressure drops of the recuperator appear in the expressions for the cycle efficiencies via $\pi_{\Delta p}^{*}$ only. It is clear that the cycle efficiency decreases with increasing pressure drops. On the other hand the solid lines in Figure 3 represent the iso-effectiveness lines for the recuperator. The effectiveness will be higher the higher the pressure drops. Based on these features for iso$\eta$ and iso- $\varepsilon$ lines, it is deduced in [12] that for the simple heat exchanger model as described in section 3.1, the optimal pressure drop ratio over the channels equals

$$
\frac{\Delta p_{c}}{\Delta p_{h}}=\sqrt{\pi_{c}} .
$$

Therefore optimal recuperator design should be searched along the set of suboptimal point indicated with a circle in Figure 3. Furthermore, based on Equation (3.6), it can be deduced [12] that

$$
\frac{n_{c}}{n_{h}}=\sqrt{\pi_{c}}, \frac{D_{h, h}}{D_{h, c}}=\sqrt{\pi_{c}} \text { and } \frac{\rho_{c}}{\rho_{h}} \approx \pi_{c} .
$$

It is clear from the discussion in Section 2.2 that all cost functions $\eta_{I I}, \eta_{W S}, \eta_{W S}^{*}$ and $\eta_{W S, 0}^{*}$ depend on $\pi_{\Delta p}^{*}$. Therefore, they all lead to the same expressions (3.6) and (3.7) that govern the suboptimal design points. Therefore we can conclude that the same line of suboptimal design points as presented in Figure 3 needs to be obtained for the other cost functions.
When the relations (3.6)-(3.7) are substituted in expression (3.5), the suboptimal $\varepsilon$ is obtained in a nondimensional form:

$$
\varepsilon=\left[1+\frac{\left(m^{*}\right)^{2} f \operatorname{Re} \operatorname{Pr}}{8 N u} \frac{\alpha_{c}\left(1+\sqrt{\pi_{c}}\right)^{3}}{\Delta p_{c}^{*} \pi_{c}^{2}}\right]^{-1},
$$

with $m^{*}=\frac{m^{\prime \prime}}{p_{0} \rho_{0}}$ the non-dimensional mass flow density. Further, since $\Delta p_{c}^{*}=\frac{\pi_{\Delta p}^{*}}{1+\sqrt{\pi_{c}}}$, this can be rewritten as:

$$
\varepsilon=\left[1+\frac{\left(m^{*}\right)^{2} f \operatorname{Re} \operatorname{Pr}}{8 N u} \frac{\alpha_{c}\left(1+\sqrt{\pi_{c}}\right)^{4}}{\pi_{\Delta p}^{*} \pi_{c}^{2}}\right]^{-1}
$$

Thus it can be concluded that for small sized recuperators $m^{*}$ is an additional recuperator design parameter.

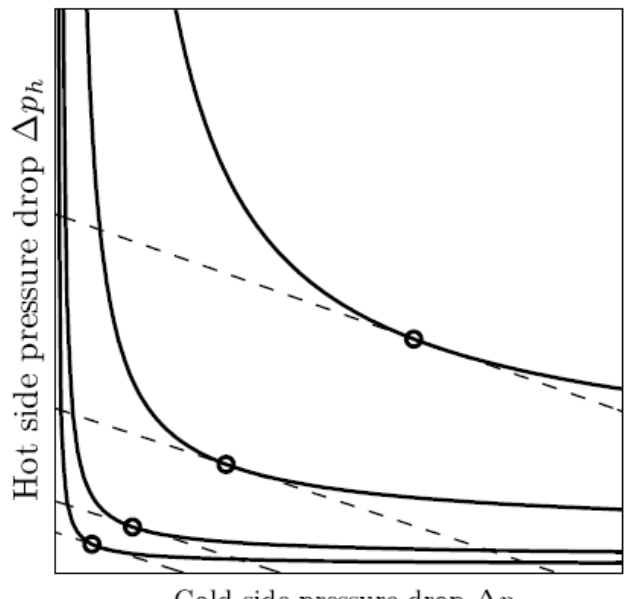

Cold side pressure drop $\Delta p_{c}$

Figure 3. Heat exchanger optimization (-- iso-efficiency lines; - iso-effectiveness lines) [12]

\section{THEORETICAL OPTIMIZATION RESULTS}

Based on expression (3.1) to (3.8) the cost functions can now be evaluated. The cost functions based on cycle efficiency $\eta_{I}$ (Equation (2.5)), the Witte-Shamsundar efficiency without and with pressure drop terms (resp. $\eta_{W S}$ and $\eta_{W S^{*}}$ in Equations (2.8) and (2.9)) as well as $\eta_{W S, 0}^{*}$ are shown in figure 4 as a function of the non-dimensional cold side pressure drop $\Delta p_{c}{ }_{c}$ of the recuperator. Data for the gas turbine cycle is based on the PowerMems project as mentioned in Section 1. This application is designed for the following operating conditions: a prescribed mass flow rate of $20 \mathrm{~g} / \mathrm{s}$ of air with $\mathrm{Pr}=0.67$, a compressor ratio of 3.3 and a turbine inlet temperature at $1200 \mathrm{~K}$. Further 


\section{Optimal micro channel recuperators for small sized gas turbines, M. Baelmans et al.}

inlet conditions $\left(1 \mathrm{~atm}, 25^{\circ} \mathrm{C}\right)$, isentropic compressor and turbine efficiencies (66 and 78\% resp.), combustion chamber parameters $\left(\pi_{c c}=0.95\right)$ and outlet pressure drops $\left(\pi_{0}=0.976\right)$ are presumed. The space given to the heat exchanger is characterized by a maximum cross section of $3,150 \mathrm{~mm}^{2}$ (6 blocks of $35 \times 15 \mathrm{~mm}^{2}$ ) and a maximal length of $100 \mathrm{~mm}$.

It can be seen that the optimal design point for $\eta_{I}$ slightly deviates from the one for $\eta_{W S}^{*}$ and $\eta_{W S, 0}^{*}$. The latter two are close to each other. The line for $\eta_{W S}$ does not lead to a maximum over the considered range of pressure drops. It should be noted that in the latter case the penalization of the pressure drop only occurs through the inlet temperature of the hot channel. Obviously the impact of recuperator pressure drops on this temperature is too weak to obtain a good approximation. On the other hand, the "decoupled" Witte-Shamsundar efficiency $\eta_{W S, 0}^{*}$ with fixed temperatures leads to a design with a relative deviation of approximately $60 \%$ in cold side pressure drop. However, due to the relative flat $\eta_{I}$-curve near its optimum, the relative error on the cycle efficiency is smaller than $2 \%$. In case the mass flow density is doubled reducing the recuperator cross sectional area to half its original size, the results as shown in Figure 5 are obtained. The optimal design point is now situated at higher pressure drops leading to smaller hydraulic diameters. Furthermore, the cycle efficiency is significantly reduced as the over-all recuperator size was decreased. The inaccuracy of the "decoupled" Witte-Shamsundar efficiency is now increased to $100 \%$ for the cold side pressure drop and a relative error on cycle efficiency of $11 \%$.

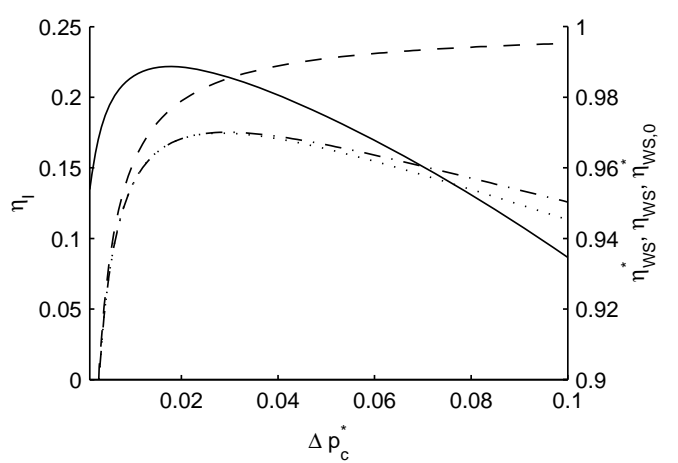

Figure 4. Suboptimal design points evaluated by cost functions for the PowerMems configuration

$$
\left(-: \eta_{I} ;-.: \eta_{W S}^{*} ;--: \eta_{W S} ; \ldots: \eta_{W S, 0}^{*}\right)
$$

In order to assess whether $\eta_{W S, 0}^{*}$ is a suitable parameter for micro channel heat exchangers and corresponding plate heat exchangers, the influence of the turbine inlet temperature, expressed by $\tau$, and of the compressor pressure ratio $\pi_{c}$ is investigated. Hereby, isentropic efficiencies and pressure drop ratios in combustion chamber and at the outlet equal to the above mentioned values. The original maximum PowerMems recuperator size is used for this computation. The relative deviation of the total pressure drop as well as the one of cycle efficiency are shown respectively in Figures 6 and 7. These deviations are respectively defined by:

$\delta \pi^{*}=\frac{\pi_{W S 0^{*}}^{*}-\pi_{I}^{*}}{\pi_{I}^{*}}$ and $\delta \eta=\frac{\eta_{W S, 0}^{*}-\eta_{I}}{\eta_{I}}$

With $\pi_{W S 0^{*}}^{*}$ the optimal $\pi^{*}$ obtained with the extended Witte-Shamsundar efficiency, neglecting the effect of recuperator pressure drops on the inlet temperatures and $\pi_{I}^{*}$ the one obtained from the full optimization based on cycle efficiency.

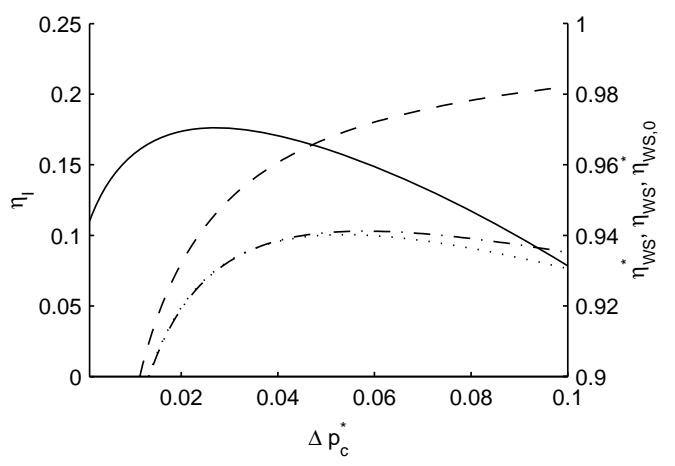

Figure 5. Suboptimal design points evaluated by cost functions for half the PowerMems configuration

$$
\left(-: \eta_{I} ;-.: \eta_{W S}^{*} ;--: \eta_{W S} ; \ldots: \eta_{W S, 0}^{*}\right)
$$

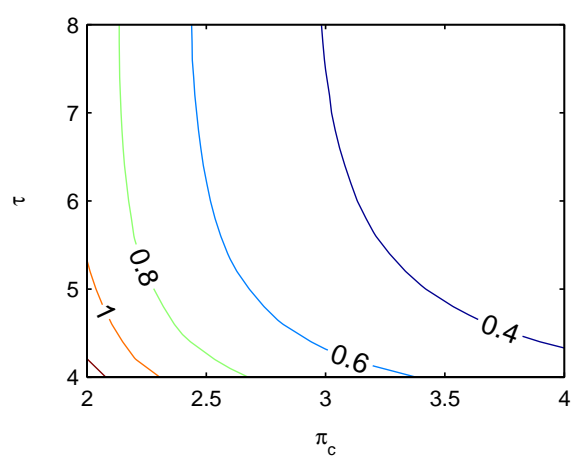

Figure 6. Relative deviation for optimal total pressure drops over the recuperator

It can be seen that the optimization via the "decoupled" Witte-Shamsundar efficiency as expressed by Equation (2.9) with fixed recuperator inlet temperatures is in general able to roughly predict the optimal recuperator geometry as long as the compressor ratio and the turbine inlet temperature do not decrease underneath the small scale PowerMems conditions $\left(\pi_{c}=3.3\right.$ and $\left.\tau \approx 4\right)$. As expected deviations become larger the smaller the pressure drop ratio over the compressor and the lower the turbine inlet temperature. 


\section{Optimal micro channel recuperators for small sized gas turbines, M. Baelmans et al.}

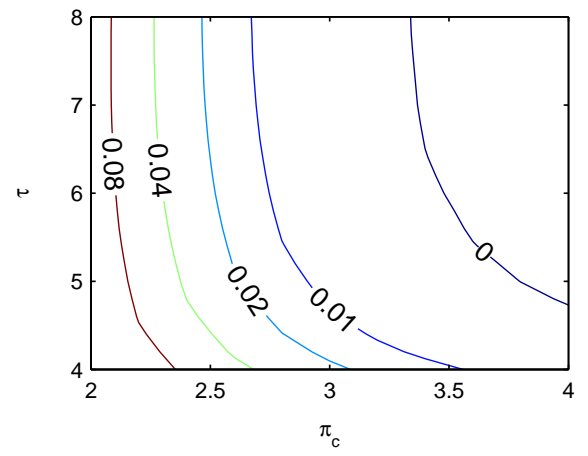

Figure 7. Relative deviations on cycle efficiencies using
the Witte-Shamsundar efficiency for optimization

\section{CONCLUSIONS}

In this paper the optimization of a recuperator for small sized gas turbines is explored. It is shown that the cycle efficiencies according to the first and second law of thermodynamics result in the same optimization points in general and to the same cost functions when the boundary for the heat input is put to infinity. Further, it is shown that the recuperator efficiency based on the definition given by Witte and Shamsundar demonstrate similar features as the cycle efficiencies, leading to the same suboptimal design points as deduced in [12]. It is shown that the non-dimensional mass flow density is a key feature in small scale heat exchanger design. Furthermore, the use of the Witte-Shamsundar efficiency is assessed and it is shown that the relative error increases for lower compressor ratios and inlet temperatures. In order to use the Witte Shansundar efficiency, the pressure term needs to be included. In addition the recuperator inlet temperatures can be fixed. With respect to design parameters for the PowerMems design, the WitteShamsundar leads to a relative deviation of less than $2 \%$ on cycle efficiency. However, the accuracy strongly depends on the mass flow density provided for the heat exchanger, which means that also the available recuperator cross-sectional area is a key parameter to assess the accuracy of the "decoupled" Witte-Shamsundar efficiency.

\section{ACKNOWLEDGEMENT}

The work of F. Rogiers is sponsored by Institute for the Promotion of Innovation through Science and Technology in Flanders (IWT-Vlaanderen).

\section{REFERENCES}

[1] L.C. Witte, N. Shamsundar, 1983, A thermodynamic efficiency concept for heat exchange devices, J. of Engineering Power, Vol. 105, pp. 199-203.
[2] Peterson, R.B., 2003, Miniature and Microscale Energy Systems, in Heat and Fluid Flow in Microscale and Nanoscale Structures, eds. M. Faghri and B. Sunden, WIT Press, London, pp. 2-15.

[3] Peirs J., Reynaerts D., Verplaetsen F., 2004, A microturbine for electric power generation, Sensors and Actuators $A$, No. 113, pp 86-93.

[4] Reynaerts D. et al., 2006, Development of a gas turbine with a $20 \mathrm{~mm}$ rotor: review and perspectives, Proc. 6th International Workshop on Micro and Nanotechnology for Power Generation and Energy Conversion Applications (PowerMEMS 2006), pp. 29-32.

[5] C.F. McDonald, 2000, Low-cost compact primary surface recuperator concept for microturbines, Applied Thermal Engineering, Vol. 20 (5), pp.471-497.

[6] C.F. McDonald \& C. Rodgers, 2008, Small recuperated ceramic microturbine demonstrator concept, Applied Thermal Engineering, 28 (1), pp. 60-74.

[7] C.F. McDonald, 2003, Recuperator considerations for future higher efficiency microturbines, 23 (12), pp. 1463-1487.

[8] S. Sullivan, X. Zhang, A.A. Ayon \& J.G. Brisson, 2001., Demonstration of a microscale heat exchanger for a silicon micro gas turbine engine, Proceedings of the International Conference on Solid-State Sensors and Actuators, Munich, Germany.

[9] E. Matsuo, H. Yoshiki, T. Nagashima \& C. Kato, 2003, Towards the development of finger-top gas turbines, Proceedings of the International Gas Turbine Congress, Tokyo, Japan, 2-7, OS-103.

[10]T. Nagasaki, R. Tokue, S. Kashima \& Y. Ito, 2003, Conceptual design of recuperator for ultramicro gas turbine, Proceedings of the International Gas Turbine Congress, Tokyo, Japan, 2-7, OS-102.

[11]T. Stevens, F. Verplaetsen, M. Baelmans, 2004, Requirements for recuperators in micro gas turbines, Proceedings of PowerMEMS Conference 2004, Kyoto, Japan, pp. 96-99.

[12]T. Stevens \& M. Baelmans, 2008, Optimal pressure drop ratio for micro recuperators in small sized gas turbines, Applied Thermal Engineering, Vol. 28, pp. 2353-2359.

[13]A. Bejan, 1996, Entropy generation minimization, CRC Press LCC.

[14]M. Yilmaz, O. Sara and S. Karsli, 2001, Performance evaluation criteria for heat exchangers based on second law analysis, Exergy an International Journal, Vol. 4, pp. 278294. 